SLAC-PUB-8876

June 2001

\title{
AN ULTRA-HIGH GRADIENT PLASMA WAKEFIELD BOOSTER*
}

\author{
P. Chen and R. Ruth \\ Stanford Linear Accelerator Center, Stanford University, CA 94309 \\ S. Cheshkov \\ Department of Physics, University of Texas, Austin, TX 78712 \\ T. Tajima \\ Department of Physics, University of Texas, Austin, TX 78712 \\ Lawrence Livermore National Laboratory, Livermore, CA 94550
}

\begin{abstract}
We present a Plasma Wakefield Acceleration (PWFA) scheme that can in principle provide an acceleration gradient above $100 \mathrm{GeV} / \mathrm{m}$, based on a reasonable modification of the existing SLAC beam parameters. We also study a possible up-grade of the Stanford Linear Collider (SLC) to hundreds of GeV center-of-mass energy using such a PWFA as a booster. The emittance degradation of the accelerated beams by the plasma wakefield focus is relatively small due to a uniform transverse distribution of the driving beam and the single stage acceleration.
\end{abstract}

Presented at the 9th Workshop On Advanced Accelerator Concepts (AAC 2000), Santa Fe, New Mexico, 6/10/2000-6/16/2001

\footnotetext{
*Work supported by the Department of Energy, contract DE-AC03-76SF00515.
} 


\section{Introduction}

Since the introduction of the plasma accelerator concepts $[1,2]$, there has been substantial progress both experimentally and theoretically that further advances the schemes [3]. Nevertheless, a macroscopic demonstration of a high gradient plasma acceleration with reasonable accelerated-beam quality, is still lacking. In the case of Laser Wakefield Accelerator (LWFA) [1], very high acceleration gradients have been observed. But the challenge has been to overcome the laser Rayleigh divergence in the plasma so as to extend the distance of acceleration. The propagation of the laser in a hollow plasma channel appears to be a promising idea [4]. On the other hand, while the electron-beam driven Plasma Wakefield Accelerator (PWFA) [2] can indeed be staged in macroscopic scale [5], the expected acceleration gradient tends to be lower than that in the LWFA scheme unless the driving beam pulse is shaped in either the linear [6, 7] or the nonlinear $[8,9]$ regime to optimize the transformer ratio $[6,7]$.

In this paper we present our study of PWFA parameters based on a reasonable extension of existing beam conditions at the Stanford Linear Accelerator Center (SLAC). We invoke the scheme of a multi-stage bunch compression that would both compress and shape the $50 \mathrm{GeV}$ SLAC beam to tens of micrometers in length. Such high density shaped beams can then excite plasma wakefields that would provide acceleration gradients of more than $100 \mathrm{GeV} / \mathrm{m}$. We also study the beam dynamics of the trailing accelerated beam, the associated beam-beam interaction effects and the luminosity deliverable. Specifically a rough design is presented for a high energy linear collider built upon adding a PWFA "booster" to the Stanford Linear Collider (SLC). We demonstrate that the collider operation at several hundred $\mathrm{GeV}$ center-of-mass energy is possible. It is interesting to note that such a "Plasma Booster" was actually proposed when the PWFA concept [10] was first introduced.

\section{Plasma Wakefields}

Our main motivation is to find a physically realizable parameter set for a linear collider application of the PWFA scheme. The fundamental principles have already been laid down when the concept was originally introduced [2, 10], and studied in 
some detail [11, 12]. For concreteness, we invoke the existing SLAC beam parameters as our starting point. Several conditions must be satisfied to use the SLAC beam as a driver. A number of assumptions are made.

First, we assume that the SLAC beams can be "bunch compressed" to a much shorter length. This is essentially the rotation of the beam in its longitudinal phase space, where the adiabatically damped relative energy spread, $\delta p / p$, is exchanged with the length of the bunch. We second assume that during bunch rotation one is able to shape the beam into an asymmetric head-to-tail density distribution for large transformer ratios, applicable to the linear regime of plasma perturbation, or a uniform distribution from head to tail for the application to the nonlinear regime. Finally, to minimize transverse focusing of the accelerated beams, we assume that the driving beam is also transversely shaped into a uniform distribution. This can in principle be accomplished by applying proper octupole magnetic fields in the beam line.

The general expressions for the longitudinal and transverse plasma wakefields are[13]

$$
\begin{aligned}
W_{\|} & =-\frac{4 \pi e n_{b}}{k_{p}^{2}} \partial_{\zeta} Z(\zeta) R(r), \\
W_{\perp} & =-\frac{4 \pi e n_{b}}{k_{p}^{2}} Z(\zeta) \partial_{r} R(r),
\end{aligned}
$$

where

$$
Z(\zeta)=k_{p} \int_{\zeta}^{\infty} d \zeta^{\prime} \rho\left(\zeta^{\prime}\right) \sin k_{p}\left(\zeta^{\prime}-\zeta\right)
$$

and $\rho(\zeta)$ is the normalized longitudinal density distribution of the driving bunch. As we assume a uniform transverse distribution, the function $R(r)$ is

$$
R(r)= \begin{cases}1-k_{p} a K_{1}\left(k_{p} a\right) I_{0}\left(k_{p} r\right), & r<a, \\ k_{p} a I_{1}\left(k_{p} a\right) K_{0}\left(k_{p} r\right), & r>a .\end{cases}
$$

Here $\zeta=z-c t$ is the beam comoving coordinate, $k_{p}=\sqrt{4 \pi r_{e} n_{p}}$ is the plasma wave number, $r_{e}=e^{2} / m c^{2}$ is the classical electron radius, $n_{p}$ is the ambient plasma density and $n_{b}$ is the beam density. $K_{i}$ 's and $I_{i}$ 's are the modified Bessel functions.

For $k_{p} a \gg 1$ and $r / a \ll 1$ we get

$$
\begin{aligned}
& W_{\|}=-\frac{4 \pi e n_{b}}{k_{p}^{2}} Z^{\prime}(\zeta)\left(1-k_{p} a \sqrt{\frac{\pi}{2 k_{p} a}} e^{-k_{p} a}\right), \\
& W_{\perp}=\frac{4 \pi e n_{b}}{k_{p}^{2}} Z(\zeta) \frac{\sqrt{2 \pi}}{4} k_{p}^{5 / 2} a^{1 / 2} e^{-k_{p} a} r .
\end{aligned}
$$


We see that the transverse wakefield is exponentially suppressed, whereas the longitudinal wakefield is slightly reduced by the form factor $F\left(k_{p} a\right)$ given by

$$
F\left(k_{p} a\right) \equiv\left(1-k_{p} a \sqrt{\frac{\pi}{2 k_{p} a}} e^{-k_{p} a}\right) \approx 1 .
$$

There is a quarter-wavelength region in the wake with simultaneous acceleration and focusing, which is the phase suitable for placing the accelerating beam.

\section{Longitudinal Bunch Shaping}

The wakefield acceleration gradient is sensitive to the longitudinal bunch shape of the driving beam. In order to search for the optimal acceleration gradient, we shall consider three representative cases of longitudinal bunch shaping that ranges from Case A: a parabola beam; Case B: a “doorstep," or optimized, beam; and Case C: a "flattop" (uniform density) beam in the nonlinear beam-plasma interaction regime. The first two cases are in the linear regime, where the plasma density is sufficiently higher than that of the beam. In Case A we intend to study the plasma wakefield generated by an unshaped, high energy beam, which is typically in Gaussian distribution. Since mathematically the parabolic density distribution is found to be easier to handle analytically than the Gaussian distribution [12], while the characteristics of the excited plasma wakefields are essentially the same, we shall invoke the parabolic, instead of the Gaussian, distribution, for Case A.

The idea is to use the existing SLC beam with compression and appropriate profile shaping as a driver for the plasma wakefield based accelerator setup in a high density plasma (or gas). This beam is characterized by an energy of $48 \mathrm{GeV}$, number of particles $N=2 \times 10^{10}$, normalized emittance $\varepsilon_{n}=3 \times 10^{-5} \mathrm{~m}$ and $\sigma_{z}=700 \mu \mathrm{m}$. However, to produce tens to hundreds of $\mathrm{GeV} / \mathrm{m}$ field we need to further shorten the bunch. Such shortening can be achieved by several bunch compression stages utilizing rotation in the longitudinal phase space of the beam. Assuming that the beam energy injected from the Damping Ring into the LINAC is $1.2 \mathrm{GeV}$, one can in principle achieve a total reduction of the bunch length by a factor of 40 when the beam reaches its final energy of $48 \mathrm{GeV}$. With the initial bunch length at $\sigma_{z}=700 \mu \mathrm{m}$, the final bunch length would 
be $\sigma_{z}=17.5 \mu \mathrm{m}$. Of course, when such a Gaussian bunch is further shaped, the total length of the beam will be different from this rms value.

Our goal is to achieve an acceleration gradient of the order of a $100 \mathrm{GeV} / \mathrm{m}$. For this purpose we choose a high plasma density. The actual plasma densities in the following three different cases will be determined by different constraints. Secondly, we wish to maximally reduce the transverse wakefield, i.e., we insist that $k_{p} a \gg 1$. Thirdly, we want to accelerate the particles over a distance of the order of $1 \mathrm{~m}$ to achieve significant final energy. When all three constraints are put together, we find it a reasonable compromise to choose $a=2 \sigma_{r}=20 \mu \mathrm{m}$, and the betatron wavelength of the beam becomes $\beta=$ $\gamma \sigma_{r}^{2} / \varepsilon_{n} \approx 30 \mathrm{~cm}$. These should allow us to meet the above requirements.

\section{Case A: Parabola (Linear Regime)}

We first examine the wakefield generated by an unshaped beam. As we have explained, it is mathematically simpler to work with a parabolic, instead of a Gaussian, distribution. In this approach, we take the half-length of the parabola, $b$, to be the $r m s$ value of the corresponding Gaussian distribution, i.e., $b=\sigma_{z}$.

The longitudinal beam density profile is given by

$$
\rho(\zeta) \equiv \frac{n_{b}(\zeta)}{n_{b}}=n_{b}\left(1-\zeta^{2} / b^{2}\right),-b \leq \zeta \leq b
$$

where $n_{b}$ is the peak density of the driving beam determined from

$$
N=n_{b} \int_{0}^{a} \int_{-b}^{b} 2 \pi r d r d \zeta\left(1-\zeta^{2} / b^{2}\right)=\frac{4}{3} n_{b} \pi a^{2} b \rightarrow n_{b}=\frac{3 N}{4 \pi a^{2} b} .
$$

In this case the longitudinal wakefield on axis behind the beam is

$$
\begin{aligned}
W_{\|} & =4 \pi e n_{b} F\left(k_{p} a\right) \int_{-b}^{b} d \zeta^{\prime}\left(1-\zeta^{\prime 2} / b^{2}\right) \cos k_{p}\left(\zeta^{\prime}-\zeta\right) \\
& =-\frac{16 \pi e n_{b}}{k_{p}^{2} b}\left[\cos k_{p} b-\frac{1}{k_{p} b} \sin k_{p} b\right] F\left(k_{p} a\right) \cos k_{p} \zeta .
\end{aligned}
$$

At locations where $k_{p} \zeta=2 n \pi$, the longitudinal wakefield $W_{\|}$reaches maxima. We define the maximum value of $\left|e W_{\|}\right|$as the acceleration gradient $G$.

We want to optimize the acceleration gradient behind the driving beam (with fixed beam parameters) by matching the bunch length with a properly chosen plasma density. This can be determined by demanding $\delta G / \delta\left(k_{p} b\right)=0$. In our case, this results in a 
choice $k_{p} b \approx 2$. Unfortunately this solution would correspond to too large a beamto-plasma density ratio, $\alpha=n_{b} / n_{p} \approx 2 / 3$, which clearly violates the assumption of linear plasma perturbation. As a compromise (but not much), we choose $k_{p} b=\pi$ so as to increase the plasma density and reduce $\alpha_{0}$. For the given $b=17.5 \mu \mathrm{m}$, we find $n_{p}=9.2 \times 10^{17} \mathrm{~cm}^{-3}$. The density ratio is now reduced to $\alpha=1 / 4 \ll 1$. With $a=20 \mu \mathrm{m}$, we have $k_{p} a=3.6$, and thus $F\left(k_{p} a\right)=0.93$. Then the acceleration gradient on the axis is

$$
G=\frac{16 \pi e^{2} n_{b}}{k_{p}^{2} b}\left[\cos k_{p} b-\frac{1}{k_{p} b} \sin k_{p} b\right] F\left(k_{p} a\right) \approx 28 \mathrm{GeV} / \mathrm{m} .
$$

We see that this acceleration gradient, though substantial, falls short of achieving the $100 \mathrm{GeV} / \mathrm{m}$ goal.

\section{Case B: “Doorstep” (Linear Regime)}

The distribution for a "doorstep" bunch is defined as

$$
n_{b}(\zeta)=\alpha_{0} n_{p}\left\{\begin{array}{lc}
1, & 0<\zeta<\frac{\pi}{2 k_{p}}, \\
1+k_{p}\left(\zeta-\frac{\pi}{2 k_{p}}\right), & \zeta>\frac{\pi}{2 k_{p}} .
\end{array}\right.
$$

Then the wake potential inside the bunch is

$$
Z^{-}=\alpha_{0} \begin{cases}1-\cos k_{p} \zeta, & 0<\zeta<\frac{\pi}{2 k_{p}}, \\ 1+k_{p}\left(\zeta-\frac{\pi}{2 k_{p}}\right), & \zeta>\frac{\pi}{2 k_{p}} .\end{cases}
$$

To find the wake potential $Z^{+}$behind the bunch, we start with the general expression

$$
Z^{+}=C_{1} \cos k_{p} \zeta+C_{2} \sin k_{p} \zeta
$$

Matching the boundary conditions at the end of the bunch $\zeta=b, Z^{-}(\zeta=b)=Z^{+}(\zeta=$ b) and $Z^{-^{\prime}}(\zeta=b)=Z^{+^{\prime}}(\zeta=b)$, we get

$$
\begin{aligned}
& C_{1}=\alpha_{0}\left\{-\sin k_{p} b+\cos k_{p} b\left[1+k_{p} b\left(1-\frac{\pi}{2 k_{p} b}\right)\right]\right\}, \\
& C_{2}=\frac{\alpha_{0}}{\cos k_{p} b}\left\{1-\sin ^{2} k_{p} b+\sin k_{p} b \cos k_{p} b\left[1+k_{p} b\left(1-\frac{\pi}{2 k_{p} b}\right)\right]\right\}
\end{aligned}
$$

An interesting special case is when $k_{p} b=n \pi$. Then

$$
\begin{aligned}
& C_{1}=\alpha_{0}\left[1+n \pi\left(1-\frac{1}{2 n}\right)\right], \\
& C_{2}=\alpha_{0} .
\end{aligned}
$$


Therefore the transformer ratio becomes

$$
R=\frac{W_{\| \max }^{+}}{W_{\| \max }^{-}}=1+n \pi\left(1-\frac{1}{2 n}\right) .
$$

The maximum acceleration gradient is

$$
G=\alpha_{0}\left[1+n \pi\left(1-\frac{1}{2 n}\right)\right] k_{p} m c^{2} F\left(k_{p} a\right) .
$$

Specifically, let us take $k_{p} b=8 \pi$. With the total length of the beam assumed to be $b=2 \sigma_{z}=35 \mu \mathrm{m}$, this corresponds to a plasma density of $n_{p}=7.3 \times 10^{18} \mathrm{~cm}^{-3}$. To

ensure self-consistency in the linear approximation of the plasma perturbation, from Eq.(12) we require that the ratio of the maximum beam density and the end of the bunch to that of the plasma be much smaller than unity. For definiteness, we set

$$
\alpha=0.5=\frac{n_{b}(\zeta=b)}{n_{p}}=\alpha_{0}\left[1+k_{p} b-\frac{\pi}{2}\right],
$$

and this fixes the parameter $\alpha_{0}=0.02$. Inserting these values into Eq.(20), we find

$$
G \approx 180 \mathrm{GeV} / \mathrm{m} .
$$

Note that this gradient is reasonably smaller than the so-called wavebreaking limit, 270 $\mathrm{GeV} / \mathrm{m}$, at the given plasma density.

\section{Case C: "Flat-Top" (Nonlinear Regime)}

Now we examine the case of a "flat-top" bunch in the nonlinear regime. By this we mean that the longitudinal density distribution of the beam is uniform from head to tail. Such a bunch distribution, though not optimized, can also provide a transformer ratio larger than 2, if the plasma density is matched to be exactly twice that of the beam[8]. One can also shape the beam in a more sophisticated manner to further optimize $R$ [9], similar to that in the linear regime[6, 7]. But for the sake of simplicity, we will consider the uniform distribution only.

Since the density of the uniform beam is $n_{b}=N / \pi a^{2} b \approx 4.5 \times 10^{17} \mathrm{~cm}^{-3}$, the matched plasma density, i.e., with $\alpha=n_{b} / n_{p}=0.5$, is $n_{p}=2 n_{b} \approx 9 \times 10^{17} \mathrm{~cm}^{-3}$, which gives $2 k_{p} b=6.3=\tau_{f}$. Using the results in [8], the maximum accelerating gradient is

$$
G=\sqrt{x-1} k_{p} m c^{2} F\left(k_{p} a\right),
$$


where $x$ is the solution to

$$
\tau_{f}=\sqrt{\chi} \sqrt{x-1}+\ln [\sqrt{x-1}+\sqrt{\chi}]
$$

Eq.(24) leads to $\chi \approx 5$ and $G \approx 166 \mathrm{GeV} / \mathrm{m}$. The transformer ratio in this case is simply

$$
R=\sqrt{\tau_{f}} \sim 2.5
$$

Comparing Case $\mathrm{B}$ and Case $\mathrm{C}$, we note that operating in the nonlinear regime has the advantage that it achieves the similar level of acceleration gradient without necessarily invoking a much higher plasma density. The price to pay, however, is that the transformer ratio in the nonlinear case is much smaller. This means with $R=2.5$, the driving beam with initial energy of $48 \mathrm{GeV}$ cannot sustain more than $L \sim 0.72 \mathrm{~m}$ in acceleration length.

The parameters discussed above are listed in Table I.

\section{Beam Dynamics and Beam-Beam Interaction Issues}

\section{Beam Dynamics Issues}

The emittance of the trailing (accelerated) bunch degrades due to the strong wakefield focusing (combined with structure errors) and binary collisions in the background plasma. To have a reasonable luminosity we need to start with a high quality beam and to deliver it to the collision point. The trailing bunch needs to be very short for two reasons - the shortness of the driver (PWFA) wavelength and to avoid big losses at the interaction point (IP) ([18]). At present it is not clear if a portion of the driver can be used as a trailing bunch due to the stringent requirements to its quality and parameters. If we use the "doorstep" scenario with $n_{p}=7.2 \cdot 10^{18} \mathrm{~cm}^{-3}$ plasma density, it gives $\lambda_{p}=13 \mu$, which makes the useful accelerating period about 3 microns. We can calculate the accelerated bunch betatron length using (“flat top” driver)

$$
\beta=\left(\frac{\gamma m c^{2}}{G \sin \Psi} \sqrt{\frac{8}{\pi k_{p} a}} \frac{e^{k_{p} a}}{k_{p}}\right)^{1 / 2} .
$$

Taking the initial beam energy $\gamma m c^{2}=1 \mathrm{GeV}, G \approx 180 \mathrm{GeV} / \mathrm{m}$ we obtain $\beta_{i} \approx$ $1 \mathrm{~cm} / \sqrt{\sin \Psi}$. If $\sigma_{z}=0.3 \mu \mathrm{m}$ then $k_{p} \sigma_{z} \approx 0.15$ so we need to take the phase at least 
TABLE I: Various bunch shapes

\begin{tabular}{|c|c|c|c|}
\hline Cases & A: Parabolc & Doorstep & Flat-Top \\
\hline \multicolumn{4}{|l|}{ Beam Parameters } \\
\hline$E[\mathrm{GeV}]$ & 48 & 48 & 48 \\
\hline$N\left[10^{10}\right]$ & 2 & 2 & 2 \\
\hline$\varepsilon_{n}\left[10^{-5} \mathrm{mrad}\right]$ & 3 & 3 & 3 \\
\hline$\sigma_{z}[\mu \mathrm{m}]$ & 17.5 & 17.5 & 17.5 \\
\hline$b[\mu \mathrm{m}]$ & 17.5 & 35 & 35 \\
\hline$\sigma_{r}[\mu \mathrm{m}]$ & 10 & 10 & 10 \\
\hline$a[\mu \mathrm{m}]$ & 20 & 20 & 20 \\
\hline$n_{b}\left[10^{17} \mathrm{~cm}^{-3}\right]$ & 2.4 & 6.4 & 4.5 \\
\hline \multicolumn{4}{|l|}{ Plasma Parameters } \\
\hline$n_{p}\left[10^{17} \mathrm{~cm}^{-3}\right]$ & 9 & 72 & 9 \\
\hline$\alpha$ & 0.25 & 0.5 & 0.5 \\
\hline$k_{p}\left[\mathrm{~cm}^{-1}\right]$ & 1800 & 5000 & 1800 \\
\hline$k_{p} a$ & 3.6 & 10 & 3.6 \\
\hline$k_{p} b$ & $\pi$ & $8 \pi$ & $2 \pi$ \\
\hline$G[\mathrm{GeV} / \mathrm{m}]$ & 28 & 180 & 167 \\
\hline$R$ & 2 & 24.5 & 2.5 \\
\hline$\beta[\mathrm{cm}]$ & 32 & 32 & 32 \\
\hline
\end{tabular}

as $\Psi=0.6$ which gives $\beta_{i} \approx 1.5 \mathrm{~cm}$. It means that even in a single stage design we need alignment control [14-16] in the submicron range to preserve the emittance of the accelerated beam (assuming initial normalized emittance of $2 \mu \mathrm{m}$, see Table II).

Following [17], we now consider the emittance growth rate from the multiple scattering in the plasma:

$$
\frac{d \varepsilon_{n}}{d z}=\frac{\gamma \beta}{2} \frac{d}{d z}<\Theta^{2}>_{p}
$$

where

$$
\frac{d}{d z}<\Theta^{2}>_{p}=\pi n_{p}\left(\frac{4 r_{e}^{2}}{\gamma^{2}}\right) \ln \left(\frac{\lambda_{D}}{R_{0}}\right),
$$

where $R_{0}=0.7 \times 10^{-13} \mathrm{~cm}$ is the effective radius of the proton and $\lambda_{D}$ is the Debye 
length $\left(\lambda_{\mathrm{D}}=k_{p}^{-1} \sqrt{k T / m c^{2}}\right)$. Assuming $\gamma=\gamma_{\mathrm{i}}+\gamma^{\prime} z$ we integrate and obtain

$$
\Delta \varepsilon_{n}=\frac{4 \pi n_{p} r_{e}^{2}}{\gamma^{\prime}} \ln \left(\frac{\lambda_{D}}{R_{0}}\right)\left(\beta_{\mathrm{f}}-\beta_{\mathrm{i}}\right),
$$

where $\beta_{\mathrm{f}}$ and $\beta_{\mathrm{i}}$ are the final and the initial betatron lengths, respectively. We used the fact that $\beta \propto \sqrt{\gamma}$. In our design the acceleration gradient is $180 \mathrm{GeV} / \mathrm{m}$ which corresponds to $\gamma^{\prime} \approx 3.5 \cdot 10^{5} \mathrm{~m}^{-1}$. In [17] the electron temperature is chosen to be 5 $\mathrm{eV}$, in our case it might be much higher but the result is very insensitive to this value. In particular for $k T=5 \mathrm{eV}$ and $n_{\mathrm{p}} \approx 7.2 \cdot 10^{18} \mathrm{~cm}^{-3}$ we obtain:

$$
\Delta \varepsilon_{n}[\mathrm{~m}] \approx 3.2 \cdot 10^{-8}\left(\beta_{\mathrm{f}}-\beta_{\mathrm{i}}\right)[\mathrm{m}],
$$

which is clearly negligible compared to the initial emittance.

\section{Beam-Beam Interaction Issues}

In the doorstep case the linear calculation gives $G \approx 180 \mathrm{GeV} / \mathrm{m}$. The required center of mass energy of $500 \mathrm{GeV}$ can be achieved in a single stage (for each arm) with a length of about $1 \mathrm{~m}$. The luminosity requirement we impose is $\mathcal{L}_{g}=10^{33} \mathrm{~cm}^{-2} \mathrm{~s}^{-1}$ which was considered in [20]. If we are to work with the SLAC driver with repetition rate of 600 $\mathrm{Hz}$, the number of particles in the accelerated bunch needs to be $5 \cdot 10^{9}$ which would cause severe beam loading issues. To study the luminosity distribution at the IP we use K. Yokoya's QED code "CAIN" [19]. The Fig. 1 shows three cases which differ by their repetition frequency at IP and the number of particles in the beam but have the same geometrical luminosity. The corresponding beam parameters: beam power $P_{b}$, particle number $N$, repetition frequency $f_{c}$, normalized emittance $\varepsilon_{n}$, IP betatron length $\beta_{x}^{*}$, IP beam transverse size $\sigma_{x}$, bunch length $\sigma_{z}$, the beamstrahlung parameter $\Upsilon$, and the relative luminosity in the last simulation bin, are listed in Table II. Clearly, high repetition frequency is necessary to reduce the number of particles required and to improve the differential luminosity (to achieve a higher peak at the design $500 \mathrm{GeV}$ center of mass energy). See Table I and Fig. 1. These results indicate the importance of studying multibunch loading in a single shot created wakefield. 


\section{Limitations and Unresolved Issues}

The primary beam is self focused (by its own wakefield), and to avoid the emittance growth due to the phase space mixing it should be matched to the focusing. However,
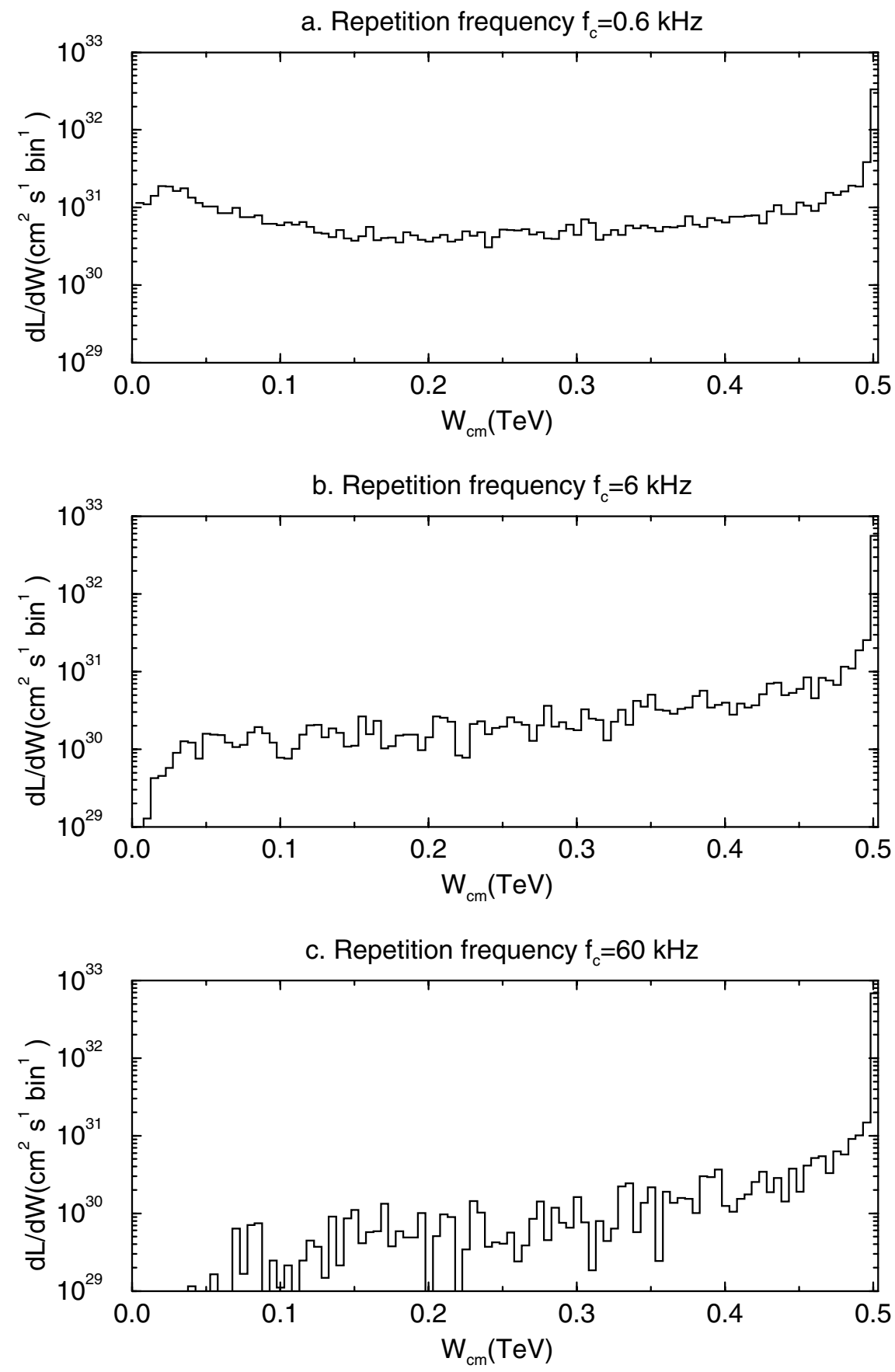

FIG. 1: Differential luminosity at repetition frequency $f=0.6,6$, and $60 \mathrm{kHz}$, respectively. The geometrical luminosity $\mathcal{L}_{g}$ was chosen $10^{33} \mathrm{~cm}^{-2} \mathrm{~s}^{-1}$ 
TABLE II: Trailing beam parameters at the IP for $500 \mathrm{GeV}, \mathcal{L}_{g}=10^{33} \mathrm{~cm}^{-2} \mathrm{~s}^{-1} e^{+} e^{-}$linear collider.

\begin{tabular}{|ccccccccc|}
$P_{b}(\mathrm{~kW})$ & $N\left(10^{9}\right)$ & $f_{c}(\mathrm{kHz})$ & $\varepsilon_{n}(\mu \mathrm{m})$ & $\beta_{x}^{*}(\mu \mathrm{m})$ & $\sigma_{x}(\mathrm{~nm})$ & $\sigma_{z}(\mu \mathrm{m})$ & $\Upsilon$ & $\frac{\mathcal{L}\left(498<W_{c m}<500 \mathrm{GeV}\right)}{\mathcal{L}_{g}}$ \\
\hline 250 & 5 & 0.6 & 2 & 30 & 11 & 0.30 & 393 & 0.4 \\
\hline 790 & 1.6 & 6 & 2 & 30 & 11 & 0.30 & 124 & 0.6 \\
\hline 2500 & 0.5 & 60 & 2 & 30 & 11 & 0.30 & 39 & 0.7
\end{tabular}

the focusing is different in the head/tail of the bunch, so there is concern associated with this.

The primary beam experiences various instabilities: transverse two-stream [7, 21], Weibel [22], electron-hose [23]. These are summarized in [3] and need investigation for the discussed scenario.

Stability of the trailing bunch also needs to be studied. It has smaller number of particles but very high density, so the loading issues might be very important.

[1] T. Tajima and J. M. Dawson, Phys. Rev. Lett. 43, 267 (1979).

[2] P. Chen, J. Dawson, R. Huff and T. Katsouleas, Phys. Rev. Lett. 54, 693 (1985).

[3] E. Esarey, P. Sprangle, J. Krall and A. Ting. IEEE Trans. Plasma Science 24, No. 2, 252 (1996).

[4] T. Chiou, T. Katsouleas, C. Decker, W. Mori, J. Wurtele, G. Shvets and J. Su, Phys. Plasmas 2, 310 (1995).

[5] M. J. Hogan et al., Physics of Plasmas 7, 2241 (2000).

[6] K. Bane, P. Chen and P. Wilson, IEEE Trans. Nucl. Sci. NS-32, 3524 (1985).

[7] P. Chen, J. Su, J. Dawson, K. Bane and P. Wilson, Phys. Rev. Lett. 56, 1252 (1986).

[8] J. Rosenzweig, Phys. Rev. Lett. 58, 555 (1987).

[9] Y. Yan and H. Chen Phys. Rev. A 38, 1490 (1988).

[10] P. Chen, R. W. Huff, and J. M. Dawson, “A Plasma Booster for Linear Accelerators", UCLA Report PPG-802, 1984.

[11] R. Ruth, A. Chao, P. Morton and P. Wilson, Part. Acc. 17, 171 (1985).

[12] R. D. Ruth and P. Chen, "Plasma Accelerators", in Supersymmetry, SLAC Report No. 296 
(1986).

[13] Pisin Chen, Part. Accelerators 20, 171 (1987).

[14] Tajima, T., Cheshkov, S., Horton, W., and Yokoya, K., in Advanced Accelerator Concepts 8, edited by W. Lawson, (AIP, New York, 1999), p.153.

[15] Cheshkov, S., Tajima, T., Horton, W., and Yokoya, K., in Advanced Accelerator Concepts 8, edited by W. Lawson, (AIP, New York 1999), p.343.

[16] Cheshkov, S., Tajima, T., Horton, W., and Yokoya, K. Phys. Rev. ST Accel. Beams 3, 071301 (2000).

[17] B. Montague, and W. Schnell, in Laser Acceleration of Particles 2 (AIP, New York, 1985) p.146 (1985).

[18] Xie, M., Tajima, T., Yokoya, K., and Chattopadhyay, S., in Advanced Accelerator Concepts 7, edited by S. Chattopadhyay, (AIP, New York, 1997), p.233.

[19] K. Yokoya, CAIN21b. http://jlcux1.kek.jp/subg/ir/Program-e.html

[20] S. Cheshkov, and T. Tajima, Int. J. Mod. Phys. A 15, 2555 (2000).

[21] T. Katsouleas, Phys. Rev. A 33, 2056 (1986).

[22] R. Keinings, and M. Jones, Phys. Fluids 30, 252 (1987).

[23] D. Whittum, W. Sharp, S. Yu, M. Lampe, and G. Joyce, Phys. Rev. Lett. 67, 991 (1991). 